\title{
Absolute and specific measures of research group excellence
}

\author{
O. Mryglod · R. Kenna • Yu. Holovatch • \\ B. Berche
}

Received: date / Accepted: date

\begin{abstract}
A desirable goal of scientific management is to introduce, if it exists, a simple and reliable way to measure the scientific excellence of publicly-funded research institutions and universities to serve as a basis for their ranking and financing. While citation-based indicators and metrics are easily accessible, they are far from being universally accepted as way to automate or inform evaluation processes or to replace evaluations based on peer review. Here we consider absolute measurements of research excellence at an amalgamated, institutional level and specific measures of research excellence as performance per head. Using biology research institutions in the UK as a test case, we examine the correlations between peer-review-based and citation-based measures of research excellence on these two scales. We find that citation-based indicators are very highly correlated with peerevaluated measures of group strength but are poorly correlated with group quality. Thus, and almost paradoxically, our analysis indicates that citation counts could possibly form a basis for deciding on how to fund research institutions but they should not be used as a basis for ranking them in terms of quality.
\end{abstract}

Keywords scientometrics $\cdot$ scientific evaluation $\cdot$ higher education

\author{
O. Mryglod \\ Institute for Condensed Matter Physics of the NAS of Ukraine, \\ 1 Svientsitskii Str., 79011 Lviv, Ukraine \\ E-mail: olesya@icmp.lviv.ua \\ R. Kenna \\ Applied Mathematics Research Centre, Coventry University, Coventry, CV1 5FB, England \\ Yu. Holovatch \\ Institute for Condensed Matter Physics of the NAS of Ukraine, \\ 1 Svientsitskii Str., 79011 Lviv, Ukraine \\ B. Berche \\ Statistical Physics Group, Institut Jean Lamour, CNRS - Nancy Université - UPVM, \\ B.P. 70239, F-54506 Vandoeuvre lés Nancy Cedex, France
}




\section{Introduction}

The problem of measuring the scientific excellence of research institutes and universities is one of current and continued importance [1. It is important to be able to detect and support the most promising research groups and to have sound and robust bases to wisely plan and invest for the future. Metrics and indicators of scientific activity may be based on the volume of researchers, the number of published papers, the amount of citations to these publications, the number of $\mathrm{PhD}$ students and on finances generated by application of scientific ideas, amongst other factors.

It is often argued that automated scientometric or bibliometric indicators may be an inadequate substitute or proxy for peer-review based evaluations of the merit of research institutes 2. There is a lot of arguments against the use of indicators in isolation, suggesting they reflect only one aspect of the research and should only be used as an adjunct to peer review (for example, see [3,1]). But in spite of the problems with indicators and metrics, reasonable and cheap approaches to the evaluation of scientific productivity and quality are desirable for practical purposes, not least because institutional peer review is an expensive and timeconsuming exercise [1.

Here we investigate "absolute" and "specific" measurements of scientific excellence on the scale of university research groups. E.g., the absolute citation count for a department is the total number of citations, irrespective of how many researchers that department contains. The corresponding specific citation count is then the average number of citations per head (see, for example, 44,5). In physics parlance these correspond respectively to the notions of "extensive" and "intensive" quantities and are usually represented mathematically by upper-case and lower-case symbols. That convention is borrowed here for absolute and specific measurements. In this paper we compare these specific and absolute notions of quality and strength with indicators of citation impact, to determine if the latter may be used as a reliable proxy for the former. We consider the overall strength of a research collective (a university department, research centre, group or some variant, component or amalgam of these) as measured through a peer-review evaluation scheme to be an absolute quantity and denote it by $\mathcal{S}$. A peer-review measure of the quality 1 s of a group of researchers is then the strength per head. Similarly, the total citation impact $\mathcal{I}$ is considered here as the absolute impact of research group while its average value $i$ (calculated per head) corresponds to specific impact. Thus, "absolute" refers to total institutional measurements while "specific" means average properties per individual. We then define strength as "volume of quality" and absolute impact as "volume of average impact".

In this paper, to make a quantitative comparison we use data from the British system of evaluation of research and education (Research Assessment Exercise) and from Evidence, a company within Thomson Reuters, one of the world's leading providers of scientific information. Using biology research groups and departments in the UK as a test case, we show that the citation-based measure $i$ is not a good proxy for the peer-review measure $s$, in that these two specific measures are rather poorly correlated. However, when scaled up to the actual size of the department $N$, the absolute citation impact $\mathcal{I}=i N$ is very strongly correlated

\footnotetext{
1 Here and further we use terms "quality" and "strength" following the notation in [6] 7
} 
with the overall strength $\mathcal{S}=s N$ as measured by peer review. (Here and below $N$ means the number of researchers in group.) This means that citations, if used in an informed manner, could possibly be used as a proxy for departmental or group strength particularly for large groups, but should not be as an estimate of research quality. When applied to large research groups or departments in particular, this may offer an alternative or at least complement to peer review.

1 Background: The research assessment exercise and the normalised citation impact

For the last quarter century, the Research Assessment Exercise (RAE) provided the premier yardstick for the measurement of scientific performance of research institutions in United Kingdom. This introduced an explicit and formalized process to assess the quality of research [8]. Six evaluation exercise have occurred since the first one in 1986 and evaluation frameworks of this type now constitute the single most important event in the UK research calender each five or so years. For the purpose of the 2008 exercise, each academic discipline was assigned to one of 67 units of assessment (UOA). In order to receive quality related funding any Higher Education Institution (HEI) could make submissions to RAE in any UOA. Using published criteria, RAE experts assess these submissions and generate graded profile for each of them. These profiles quantify the proportion of a department's or research centre's work which falls into each of five quality bands as follows [8]:

$-4^{*}$ : Quality that is world-leading in terms of originality, significance and rigour;

$-3^{*}$ : Quality that is internationally excellent in terms of originality, significance and rigour but which falls short of the highest standards of excellence;

$-2^{*}$ : Quality that is recognised internationally in terms of originality, significance and rigour;

- 1*: Quality that is recognised nationally in terms of originality, significance and rigour;

- Unclassified - Quality that falls below the standard of nationally recognised work or work which does not meet the published definition of research for the purposes of the assessment.

An example of a quality profile is given in Table 1

Besides obvious benefits in terms of prestige, marketing and publicity potential, the research quality profiles determined how much funding each university department receives in the years following the exercise. Funding is determined by a formula combining the weighted quality scores of individual research group or centres. The formula is subject to regional and temporal variation, but the one introduced by the Higher Education Funding Council for England (HEFCE) immediately subsequent to RAE 2008 was [10]

$$
s=p_{4 *}+\frac{3}{7} p_{3 *}+\frac{1}{7} p_{2 *}
$$

Here $p_{n *}$ represents the percentage of a team's research which was rated $n *$ and $s$ may be considered as a single specific measure of a university's overall research quality in a particular discipline. The total amount of quality related funding 
distributed by HEFCE to a given university post RAE is proportional to the overall strength of the submission

$$
\mathcal{S}=s N
$$

where $N$ is the number of researchers submitted from a given university. Thus, strength $\mathcal{S}$ is an absolute measure of performance.

Notwithstanding various managerial tactics and manoeuvres, the RAE, and similar exercises in other countries, is considered to be reasonably reliable since it is based on peer-review evaluation. Despite its many drawbacks and limitations, peer review is considered by the academic community at large to be the most reliable and trustworthy scheme to evaluate the worth of curiosity-driven research, in particular. On the other hand, peer-review-based evaluation is expensive and time-consuming process. In addition, the very act of measuring the scientific system distorts the very process it purports to measure. This is Goodhart's Law, a type of socioeconomic counterpart of the Heisenberg uncertainty principle in physics. Therefore, it is reasonable to propose some alternatives to the RAE. Evaluations based entirely upon citation counts are some of many possible candidate schemes (see, for example, [11,12). Some authors advocate substitution of the RAE by citation counting due to claimed good correlation between the resultant rankings obtained [11,13. But there are also authors (e.g., [14) who point to different weaknesses of citation analysis such as self-citing, bias, technical errors connected with citation data retrieval or different typical rates of citations for various disciplines. Although methods have been, and are being, developed to try to deal with these phenomena, no universally satisfactory automated system has yet emerged.

So, the question arises as to how good citation-based indicators can be as proxies for peer review. Since the last UK exercise generated quality measurements in far more detail than previous RAEs, and since subsequent evaluation frameworks in the UK will amalgamate some of the UOA's used in 2008 into fewer, larger subject groupings, RAE 2008 provides a window of great detail in which answers to this question may be sought. Having precise numbers assigned to each institution for the 67 disciplines it is possible to perform accurate comparisons between RAE

Table 1 An example of quality profile of RAE 2008 for the biology department of Open University ${ }^{\ddagger}$.

\begin{tabular}{|l|l|l|l|l|l|}
\hline & \multicolumn{5}{|c|}{$\begin{array}{c}\text { Quality profile } \\
\text { (percentage of research } \\
\text { activity at each quality level) }\end{array}$} \\
\hline & $4^{*}$ & $3^{*}$ & $2^{*}$ & $1^{*}$ & unclassified \\
\hline \hline Output & 5.3 & 17.7 & 46.1 & 23.7 & 7.2 \\
\hline Environment & 0.0 & 14.7 & 50.9 & 33.2 & 1.2 \\
\hline Esteem & 7.1 & 42.5 & 41.5 & 3.6 & 5.3 \\
\hline Overall & 5.0 & 20.0 & 45.0 & 25.0 & 5.0 \\
\hline
\end{tabular}

\footnotetext{
$¥$ An overall quality profile is constructed by summing the three separate weighted components ("Output", "Environment" and "Esteem") using a special cumulative rounding methodology which avoids unfair consequences that simple rounding can produce 9 .
} 
peer-review evaluation and citation scores. Thus we arrive at the main questions we wish to address herein: how do RAE 2008 scores correlate with citation rates?

As already noticed before, to get the citation-based measure, we use the data provided by Evidence. This company offers a service analysing research performance tailored to individual client requirements [15]. We consider the so-called normalised citation impact (NCI) $i$ used by Evidence as a coefficient of departmental performance in a given discipline.

Evidence calculate the NCI using data from Thomson Reuters databases [3, 16]. Similarly to Relative Citation Rate (RCR) (i.e., [17), NCI is a relative measure as it is calculated by comparing to a mean or expected citation rate. On the other hand, this is a specific measure of citation impact because it is averaged to be a measure of impact for research group. It has long been known that citation rates are different for different disciplines. The main advantage of Evidence calculations is non-trivial normalisation of citation counts between different academic disciplines. To achieve this, the total citation count for each paper can be normalised to an average citations per paper for the year of publication and either the field or journal in which the paper was published (the so-called "rebasing" of the citation count). The normalised value is known as the NCI [16]. Only the four papers per individual which were submitted to RAE 2008 were taken into account by Evidence in order to calculate the average NCI for research groups [16]. The RAE does not emplot a comparable normalisation process and this is a serious and acknowledged weakness of that peer evaluation exercise [18.

Thus, the NCI may be considered as a specific measure of the research impact of a department in a given field and we denote it by $i$. The corresponding absolute measure of impact (the total impact of the department or group) is denoted by $\mathcal{I}$. The relationship between the two is

$$
\mathcal{I}=i N
$$

In the following, we compare the specific indicators of quality and impact $s$ and $i$ as measures of the average strength and impact of the group or department per individual contained within it. We also compare the counterpart absolute measurements $\mathcal{S}$ and $\mathcal{I}$ as measures of the overall strength and total impact of the group as a whole. We use the biology research sector in the UK as a test case. We show that while the specific measures $s$ and $i$ are only weakly correlated, the absolute ones $\mathcal{S}$ and $\mathcal{I}$ are strongly aligned. The analysis thus suggests that citations form a poor basis for measuring institutional research quality but could form a good basis for measuring strength. Since quality related funding as defined by HEFCE is proportional to departmental strength rather than quality, the citation-based measures may offer a reasonable and cost effective way to decide on funding while ameliorating the negative effects of Goodhart's law.

We have to note that although RAE assessments as well as the Evidence results are used for practical purposes, they are still subject to debate within the scientometric community. This is an additional reason to compare them.

\section{Weak correlation between specific measures of quality and impact}

If the NCI score formed a perfect proxy for RAE peer-review quality measures, there would be a perfect linear correlation between $i$ and $s$. The actual correlation 
is depicted in Fig. 1 where $i$ is plotted against $s$ for biology research groups. The relevant data are contained in Table 2, where the RAE measured qualities and strengths of different institutions are listed alongside specific and absolute NCI values. The quality values $s$ come from RAE 2008 and the impact scores from Ref. [3]. While a general alignment is evident (groups with high RAE quality scores tend to have high NCI measures) there is considerable scatter. This is quantified

Table 2 The ranking of UK biology departments using the RAE 2008 scores $s$ and the NCIbased scores $i$.

\begin{tabular}{|c|c|c|c|c|}
\hline HEI & $\begin{array}{l}\text { Average } \\
\text { quality, } s\end{array}$ & $\begin{array}{l}\text { Ranking } \\
\text { by } s\end{array}$ & $\begin{array}{l}\text { Average } \\
\text { NCI, } i\end{array}$ & $\begin{array}{l}\text { Ranking } \\
\text { by } i\end{array}$ \\
\hline Institute of Cancer Research & 61.43 & 1 & 2.26 & 1 \\
\hline University of Manchester & 46.43 & 2 & 1.32 & 14 \\
\hline University of Dundee & 45.71 & 3 & 2.06 & 2 \\
\hline University of Sheffield & 45.00 & 4 & 1.25 & 17 \\
\hline University of York & 44.29 & 5 & 1.67 & 5 \\
\hline Imperial College London & 42.86 & 6.5 & 1.56 & 7 \\
\hline King's College London & 42.86 & 6.5 & 1.45 & 10 \\
\hline Royal Holloway. University of London & 42.14 & 8 & 1.05 & 31 \\
\hline University of Cambridge & 41.43 & 9 & 1.85 & 3 \\
\hline University of Leeds & 39.29 & 10 & 1.32 & 15 \\
\hline University of Edinburgh & 38.57 & 11.5 & 1.53 & 8 \\
\hline University of Newcastle upon Tyne & 38.57 & 11.5 & 1.20 & 22 \\
\hline University of Glasgow & 37.14 & 14 & 1.23 & 19 \\
\hline Cardiff University & 37.14 & 14 & 1.18 & 24 \\
\hline University of Aberdeen & 37.14 & 14 & 1.02 & 34 \\
\hline University of St Andrews & 33.57 & 16 & 1.13 & 26 \\
\hline University of Bath & 32.86 & 19.5 & 1.34 & 13 \\
\hline University of Durham & 32.86 & 19.5 & 1.27 & 16 \\
\hline University of Birmingham & 32.86 & 19.5 & 1.25 & 18 \\
\hline University of Nottingham & 32.86 & 19.5 & 1.13 & 27 \\
\hline University of East Anglia & 32.86 & 19.5 & 1.09 & 29 \\
\hline University of Exeter & 32.86 & 19.5 & 0.84 & 38 \\
\hline University of Southampton & 32.14 & 23.5 & 1.15 & 25 \\
\hline University of Warwick & 32.14 & 23.5 & 1.09 & 28 \\
\hline University of Leicester & 30.71 & 25 & 1.63 & 6 \\
\hline University of Liverpool & 29.29 & 26 & 1.04 & 32 \\
\hline University of Essex & 26.43 & 27.5 & 1.46 & 9 \\
\hline Queen Mary. University of London & 26.43 & 27.5 & 1.19 & 23 \\
\hline University of Sussex & 24.29 & 29.5 & 1.75 & 4 \\
\hline University of Reading & 24.29 & 29.5 & 1.04 & 33 \\
\hline University of Kent & 23.57 & 31 & 0.88 & 35 \\
\hline Queen's University Belfast & 22.86 & 32 & 0.82 & 39 \\
\hline Bangor University & 21.43 & 33 & 0.84 & 37 \\
\hline Open University & 20.00 & 34 & 0.88 & 36 \\
\hline Oxford Brookes University & 17.86 & 35.5 & 1.22 & 21 \\
\hline University of Plymouth & 17.86 & 35.5 & 0.64 & 42 \\
\hline University of Hull & 17.14 & 37 & 1.43 & 11 \\
\hline Cranfield University & 16.43 & 38.5 & 1.07 & 30 \\
\hline Swansea University & 16.43 & 38.5 & 0.67 & 41 \\
\hline University of Derby & 11.43 & 40.5 & 1.41 & 12 \\
\hline Liverpool John Moores University & 11.43 & 40.5 & 0.75 & 40 \\
\hline University of Glamorgan & 8.57 & 42 & 0.36 & 43 \\
\hline Roehampton University & 7.14 & 43 & 0.36 & 44 \\
\hline Bath Spa University & 2.14 & 44 & 1.23 & 20 \\
\hline
\end{tabular}




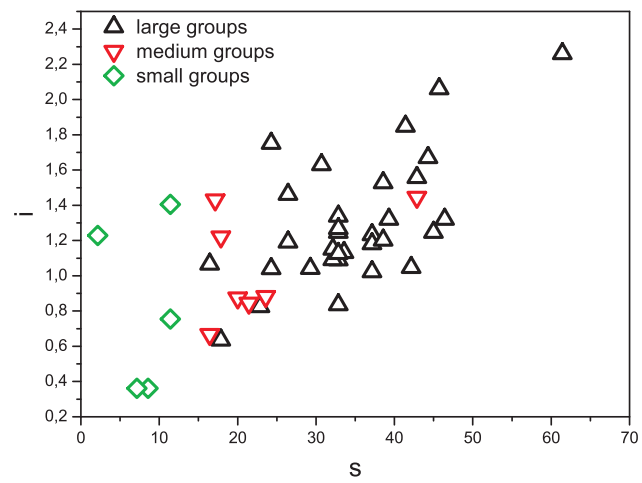

Fig. 1 Correlation between $s$ (average quality of research groups according to RAE 2008) and $i$ (average excellence of research groups according to normalised citation impact).

by a relatively small value of the Pearson coefficient of $64 \%$. (Table 3 contains a full list of Pearson correlation coefficients measured in this paper.)

In Refs. 6, 7], a quantitative analysis of the dependency of group or departmental research quality on size was given. This allowed the determination of two types of critical mass in research. It was shown that, for a multitude of different academic disciplines, there is a linear relationship between quality and quantity up to a certain group size, known as the upper critical mass $N_{c}$. At this point coordination problems set in and a phenomenon similar to the Ringelmann effect 2 ensures that groups of size greater than $N_{c}$ have either a reduced dependency of quality on quantity or no such dependency. In Refs. 6] 7] a lower critical mass $N_{k}$ was also defined and measured for many disciplines. This was interpreted as the minimum size a research department should achieve to be stable in the long term. The two critical masses, the values of which are strongly dependent on the research discipline, allow research groups and departments to be categorised as being small if they have size $N \leqslant N_{k}$, medium if $N_{k} \leqslant N \leqslant N_{c}$ or large if $N>N_{c}$. For the biology UOA, the estimates for critical masses are $N_{k}=10.4$ and $N_{c}=20.86$, 7. (Fractions of staff are a feature of RAE in that HEI's can include part-time researchers in their submissions. These are counted as a proportion of full time equivalence [8].)

\subsection{Weak correlation between specific measures of overall quality and impact}

The question arises whether citation counts could serve as a proxy for RAE scores for small, medium or large groups separately. In Fig. 1 the small, medium and large research groups are distinguished by different colors. While there is considerable scatter across all three categories, it is most pronounced for small and medium groups, an observation which is confirmed by the values of Pearson coefficients calculated separately for small $(r<0)$, medium $(r \approx 47 \%)$ and large groups $(r \approx 62 \%$ ) (Table 3). Nonetheless, it provokes the question of whether size is

2 The Ringelmann effect describes the tendency for average productivity to reduce as the size of the group increases, while in Refs. 66,7] a reduction in the "rate of change" of quality with quantity is observed. 


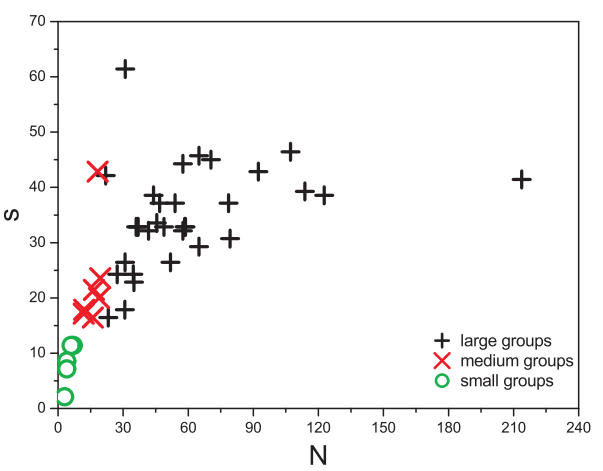

(a)

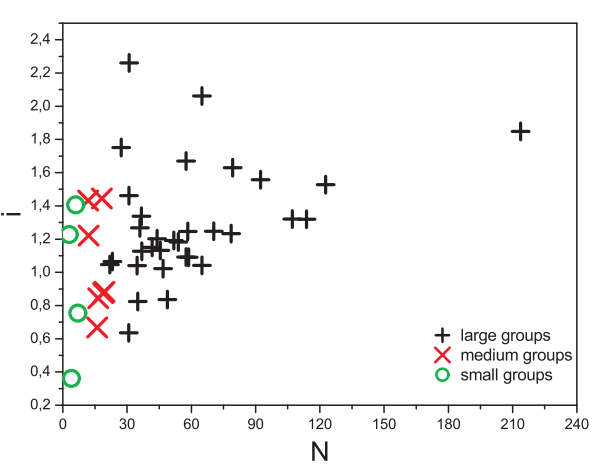

(b)

Fig. 2 The average excellence of research groups as a function of group size $N$ for biology according to: (a) RAE 2008 assessments and (b) normalised citation impact.

a relevant feature which should be taken into account in attempting to build a citation-based proxy for peer review.

The relationship between RAE-measured quality $s$ and quantity $N$ for biology is depicted in Fig. 2(a) (see also Refs. 6,7]). The counterpart relationship between specific impact $i$ and quantity $N$ is shown in Fig. 2(b) (see also Ref. [3]). Clearly, both quality and impact are correlated with quantity and the plots exhibit similar features. A striking similarity is apparent between both plots: they each have a distinct maximum and each have no data in the bottom right regions, reflecting the fact that there are no low quality, or low impact large biology research groups in the UK. On the other hand, the plots differ significantly in much of the details, especially in the region of small and medium groups.

2.2 Weak correlation between ranked measures of overall quality and impact

An increasingly common phenomenon associated with any attempt to measure scientific performance is the ranking by various media of different institutions according to their perceived quality or impact. Although overly simplistic, such tables are frequently reported used to inform potential students of the standing of various universities. In Ref. [6] it was shown that such systems are inherently dangerous if used to compare research quality because they do not properly take size and resources into account.

Earlier analyses attempted to build a citation-based proxy for peer review using such rankings. Because earlier renditions of the RAE supplied as outcome a single number (rather than a profile) purporting to encapsulate the research performance of a department, much less information was available than we have access to in RAE 2008. In those cases, correlations were sought not between absolute RAE scores and bibliometrics, but between rankings resulting form such scores [11,12]. We therefore also checked for possible correlations between the ranked values of $s$ and $i$. In order to build the ratings of research groups they should be listed in ascending order of their corresponding scores. Each department is assigned an ascending numerical rank (the average rank in the case of the equal scores) in Table 2. The calculated value of the Spearman rank-order correlation coefficient 


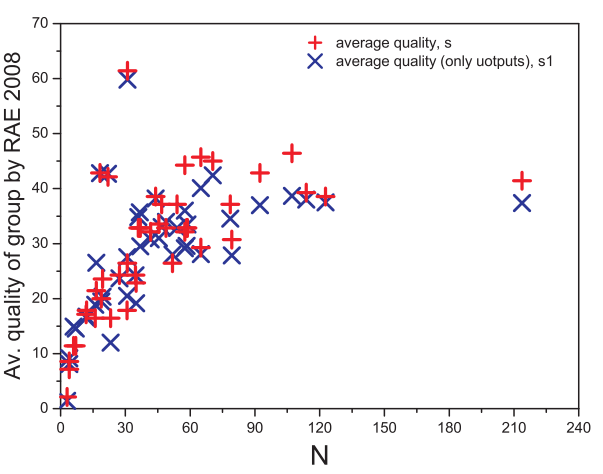

(a)

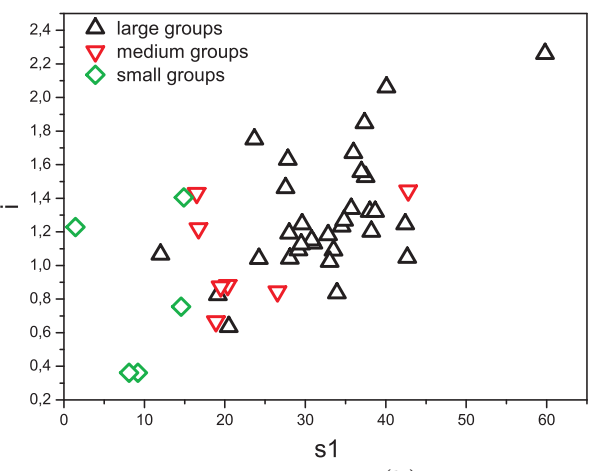

(b)

Fig. 3 (a) The average excellence of research groups as a function of group size $N$ for biology according to RAE 2008 assessments. Here the overall quality $s$ corresponds to the symbols + , while output measures $s_{1}$ are denoted by $\times$. (b) Correlation between $s_{1}$ ("outputs" according to RAE 2008) and $i$ (from NCI scores).

is approximately equal to $57 \%$ (Table 3) and is even worse than the correlations based on Fig. 1.

Therefore, in contrast to earlier claims in Refs. [11, 12, we do not observe strong correlation between rankings of HEI's according to RAE and to the citation-based measure. Instead, only weak linear correlation could be observed for ranked values of $s$ and $i$.

2.3 Weak correlation between specific measures of elemental quality and impact

At RAE, three elements of research quality were examined to arrive at an overall quality profile, namely research outputs, research environment and research esteem. For the biology UOA, to arrive at the overall quality score, these three elements were weighted with $75 \%$ of the overall score coming from the perceived quality of outputs (mostly publications), 20\% coming from environment and $5 \%$ attributable to esteem (see Table 1). Thus, only $75 \%$ of overall RAE estimations correspond to evaluation of published papers. Citation counts, in contrast, rely $100 \%$ on the quality of papers. Since Evidence use only the citation data to calculate NCI, we may suppose it is more sensible to compare these results with only the output element or RAE. The overall quality profile, and sub-profiles for research outputs, research environment and esteem indicators for each submission are available on the official RAE web-page [19]. Here separate profile values for "Outputs", "Environment" and "Esteem" could be used instead of the overall values. Hence, the separate components of the average quality could be calculated using the same funding formula (11). We denote by $s_{1}, s_{2}$ and $s_{3}$ the quality measure coming from outputs, environment and esteem, respectively.

Differences between $s$ and $s_{1}$ values are observable in Fig. 3 (a) where they are both plotted. Obviously, however, the overall average quality scores $s$ and those coming from outputs only $s_{1}$ are quite close to each other, since the contribution of $s_{1}$ into $s$ is $75 \%$. It is interesting to observe that the overall quality score $s$ is mainly greater than the output score $s_{1}$ for large groups (see Fig. 3). This indicates that 


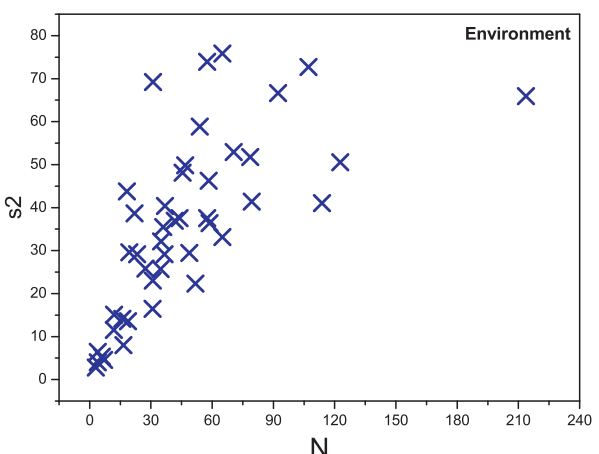

(a)

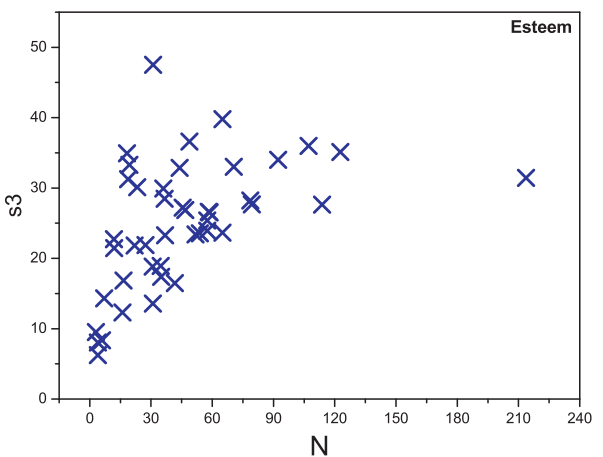

(b)

Fig. 4 (a) Correlation between $s_{2}$ ("Environment" component of average quality of research groups according to RAE 2008) and $N$. (b) Correlation between $s_{3}$ ("Esteem" component of average quality of research groups according to RAE 2008) and $N$.

the RAE evaluators saw added benefit in large-scale research departments. Another explanation is the extra visibility that large groups enjoy. Small and medium groups are disadvantaged in these respects - they have neither the visibility nor all of the facilities available to their larger competitors.

The relationships between the "environment" $\left(s_{2}\right)$ and "esteem" $\left(s_{2}\right)$ components of RAE quality profiles and group size $N$ are shown in Fig. 4. One observes that $s_{2}$ is more linearly correlated with $N$ than the other measures $s_{1}$ and $s_{3}$. This reflects the fact that bigger research groups have access to more expensive and more complex equipment. Economy of scale thus ensures that the average fraction of "environment" continues to grow with group size, even for very large groups or departments.

Nonetheless, the linear correlation between $s_{1}$ and $i$ is not stronger than that between $s$ and $i$. In fact, the corresponding Pearson coefficient at $53 \%$ is even smaller (Table 3). Moreover, there is the same high degree of scatter for small and several medium groups. To be comprehensive, we have also determined the correlation coefficients for the cases of environment quality $s_{2}$ and esteem $s_{3}$. The resulting correlation coefficients are $r=55 \%$ and $r=46 \%$, respectively. In conclusion, there is only weak correlation between Evidence indicators of impact and RAE 2008 scores for average quality of research groups. Therefore it is not possible to use the NCI as a direct proxy for peer review measures of academic research quality.

\section{Strong correlation between absolute measures of research strength and impact}

A conclusion of the above analysis is that there is only weak correlation between the specific measure of impact $i$ and the RAE peer-evaluated measure of quality $s$, and that this is especially weak, or absent for small groups. Possible reasons for this include the facts that (i) there are few small and medium groups (only 5 small groups and only only 7 medium groups) in biology and (ii) small groups, by definition, have relatively few members. Both of these factors mean that the 
statistics for small (and to a lesser extent medium) groups are relatively poor in comparison to those for large groups. Since the number of large groups is bigger and also the statistics for large groups are better, more robust results can be obtained for large groups.

Moreover, in the above analysis, all research groups are treated as contributing the same weight to the analysis. E.g., the three researchers from Bath Spa University contribute to the same extent as the 213.69 biologists from Cambridge University! It is therefore sensible to correct this anomaly by introducing weights. Multiplying the average quality of groups by their size renders the specific measures absolute: quality becomes strength and the NCI is also scaled up to the volume of the group or department.

The RAE, peer-reviewed measures of strength $\mathcal{S}$ are compared to the absolute, citation-based measures of NCI in Fig. 5. The correlation between the two measures is impressive. This is reflected in the almost perfect Person correlation coefficient $r=97 \%$ (see Table 3). Moreover, restricting the analysis only to large groups also reveals an excellent correlation of $96 \%$. The correlation coefficients for medium groups alone is less good, at $86 \%$ and for small groups that figure is $65 \%$. However, since small and medium research groups tend to have the same linear dependency of quality on quantity [6 it is more sensible to combine them in the correlation analysis. Indeed, the Pearson correlation coefficient for small and medium groups combined is $92 \%$. Thus we can say that the NCI forms a good proxy for peerreview estimates of group research strength for large and small/medium research

Table 3 The values of correlation coefficients calculated for different data sets.

\begin{tabular}{|c|c|c|}
\hline $\begin{array}{l}\text { Description of the } \\
\text { data sets }\end{array}$ & $\begin{array}{l}\text { Pearson coefficient } r \text { and } P \text {-value } \\
\text { comparing to significance level } \alpha=0.05^{\dagger}\end{array}$ & $\begin{array}{l}\text { Spearman } \\
\text { coefficient }\end{array}$ \\
\hline$s$ vs $i$ & $\begin{array}{l}\approx \approx 0.64, P<\alpha \\
\text { (large groups) } r \approx 0.62, P<\alpha \\
\text { (medium groups) } r \approx 0.47, P>\alpha \\
\text { (small groups) } r<0, P>\alpha \\
\text { (small \& medium groups) } r \approx 0.39, P>\alpha\end{array}$ & 0.57 \\
\hline$s_{1}$ (only outputs) vs $i$ & $\begin{array}{l}r \approx 0.60, P<\alpha \\
\text { (large groups) } r \approx 0.57, P<\alpha \\
\text { (medium groups) } r \approx 0.36, P>\alpha \\
\text { (small groups) } r \approx 0.03, P>\alpha \\
\text { (small \& medium groups) } r \approx 0.35, P>\alpha\end{array}$ & 0.53 \\
\hline$s_{2}$ (only environment) vs $i$ & $\begin{array}{l}r \approx 0.64, P<\alpha \\
\text { (large groups) } r \approx 0.63, P<\alpha \\
\text { (medium groups) } r \approx 0.40, P>\alpha \\
\text { (small groups) } r<0, P>\alpha \\
\text { (small \& medium groups) } r \approx 0.36, P>\alpha\end{array}$ & 0.55 \\
\hline$s_{3}($ only esteem) vs $i$ & $\begin{array}{l}r \approx 0.58, P<\alpha \\
\text { (large groups) } r \approx 0.54, P<\alpha \\
\text { (medium groups) } r \approx 0.40, P>\alpha \\
\text { (small groups) } r \approx 0.20, P>\alpha \\
\text { (small \& medium groups) } r \approx 0.41, P>\alpha\end{array}$ & 0.46 \\
\hline $\mathcal{S}$ vs $\mathcal{I}$ & $\begin{array}{l}r \approx 0.97, P<\alpha \\
\text { (large groups) } r \approx 0.96, P<\alpha \\
\text { (medium groups) } r \approx 0.86, P<\alpha \\
\text { (small groups) } r \approx 0.65, P>\alpha \\
\text { (small \& medium groups) } r \approx 0.92, P<\alpha\end{array}$ & - \\
\hline
\end{tabular}

$\dagger$ if $P<\alpha$ then the linear correlation is considered as statistically significant 


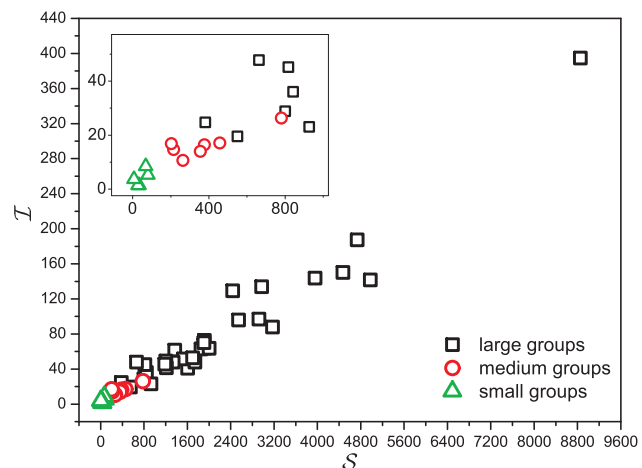

Fig. 5 Correlation between absolute quality values (strength) for research groups according to RAE 2008 and normalised citation impact results (the region of small and medium groups on the inset).

departments. Fig. 5 should be compared to Fig. 1. The replacement of specific measures of quality and impact by their absolute counterparts has had the effect of stretching the corresponding axes by an amount proportional to the quantity of the groups or departments. Because of the clear relationship between quality and quantity identified in Ref. [6,7], and a similar relationship between NCI impact and quantity observed in Ref. [3], this stretching induces the improved correlations observed.

\section{Conclusions}

A goal of scientometrics is to develop a method to provide a reliable measurement of scientific excellence using minimal efforts. However, any attempts to replace current peer-evaluation based systems have to be done carefully and robustly. Quality related funding, such as that administered by HEFCE post RAE in the UK is proportional to absolute measures of research strength since financial support for research obviously needs to be weighted according to group size. Therefore, a citation based approach, at least for large groups, may be introduced as less intrusive and more cost effective alternative to national peer review. This would also have the advantage of ameliorating the distortions to the research system introduced on a national basis every five years or so through Goodhart's phenomenon, although one might expect a citation-based system to introduce distortions of its own.

However, we have also seen that the citation metrics used here are not well correlated with peer-review measures of group research excellence. Since the latter are used to rank institutions, it is clear that citation counts are not a good basis on which to make such comparisons.

Thus we arrive at the almost paradoxical conclusion that the citation-based metric (NCI) may be used as an excellent proxy for peer-reviewed measurements of institutional scientific strength but it is only a poor proxy for measures of quality. Since quality related funding is strength based, this may offer a much cheaper alternative to the system currently in use in the UK and some other countries.

The analysis presented here is based on biology research groups in the UK. Further analyses are underway for other disciplines to determine the broader suit- 
ability of scientometric measures of specific and absolute research-group properties. The nuances connected with peculiarities of groups of different sizes should be also studied. Since in the UK system, a large group with, say 40 members, provides $40 \times 4=160$ submissions, which may be sufficiently large for statistical fluctuations to be ironed out. But the RAE evaluation of a small group, say with 5 staff members, is based on the statistics of only approximately $5 \times 4=20$ submissions. This is far more susceptible to inaccuracies and statistical noise. So, any automated evaluation of large groups is much more robust than for small groups even if one is only interested in measuring strength rather than quality. A possible way to balance this, if introducing citation counts, would be to keep peer review for small and medium sized groups or to require a greater number of outputs per person for such groups to improve statistics. Investigations into such schemes are underway. In any case, it is clear from the current test case that any attempt to automatically evaluate research quality should very carefully take account of group or departmental sizes [6].

\section{Acknowledgements}

This work was supported in part by the 7th FP, IRSES project No. 269139 "Dynamics and cooperative phenomena in complex physical and biological environments" and IRSES project No. 295302 "Statistical physics in diverse realizations". The authors thank Jonathan Adams from Evidence for the data and Ihor Mryglod for fruitful discussions.

\section{References}

1. De Bellis N. Bibliometrics and Citation Analysis: From the Science Citation Index to Cybermetrics. Scarecrow Press (2009).

2. Editorial, Nature, 2010, 465 p.845 and Metrics Special at www.nature.com/metrics (last accessed April 2012).

3. The future of the UK university research base. Evidence (a Thomson Reuters business) report, July 2010.

4. Vinkler P. An attempt for defining some basic categories of scientometrics and classifying the indicators of evaluative scientometrics. Scientometrics (2001), 50, No. 3, 539-544.

5. Vinkler P. Relations of relative scientometric indicators. Scientometrics (2003), 58, No. 3, 687-694.

6. Kenna R., Berche B., Critical mass and the dependency of research quality on group size, Scientometrics, 2010, 86, No. 2, 527-540.

7. Kenna R., Berche B., Critical masses for academic research groups and consequences for higher education research policy and management, Higher Education Management and Policy, 2011, 23, Iss. 3, 1-21.

8. The official web-page of the RAE 2008: http://www.rae.ac.uk/

9. The panel criteria and working methods, available on the otticial web-page of RAE 2008: http://www.rae.ac.uk/panels/main/d/bio/

10. The official web-page of the Higher Education Funding Council for England: http://www.hefce.ac.uk/

11. Oppenheim Ch., Do citations count? Citation indexing and the Research Assessment Exercise(RAE), Serials: The Journal for the Serials Community, 1996, 9, No. 2, 155-161.

12. Norris M., Oppenheim Ch., Citation counts and the Research Assessment Exercise. V Archaeology and the 2001 RAE, Journal of Documentation, 2003, 59, No. 6, 709-730.

13. Holmes A., Oppenheim C. Use of citation analysis to predict the outcome of the 2001 Research Assessment Exercise for unit of assessment (UoA) 61: library and information management. Information Research, (2001), 6(2). Retrieved from http://informationr.net/ir/6-2/paper103.html 
14. MacRoberts M.H., MacRoberts B.R. Problems of citation analysis: a critical review. Journal of the American Society for Information Science (1989), 40(5), 342-349.

15. The official web-page of Evidence Thomson Reuters: http://www.evidence.co.uk

16. Funding research excellence: research group size, critical mass \& performance. A University Alliance report, July 2011.

17. Schubert A., Braun T. Cross-field normalization of scientometric indicators. Scientometrics (1996), 36, No. 3, 311-324.

18. Kenna R., Berche B., Normalization of research evaluation results across academic disciplines, Research evaluation 20 (2011) 107-116.

19. The overall quality profile, and sub-profiles for research outputs, research environment and esteem indicators for each submission for RAE 2008 could be found here: http://www.rae.ac.uk/pubs/2009/pro/Quality_profiles.xls 